\title{
Integrated gene network analysis and text mining revealing PIK3R1 regulated by miR-127 in human bladder cancer
}

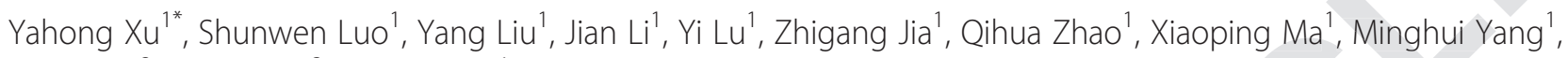
Yue Zhao ${ }^{2}$, Ping Chen ${ }^{3}$ and Yu Guo ${ }^{1}$

\begin{abstract}
Background: Cancer is the result of a complex multistep process that involves the accumulation of sequential alterations of several genes, including those encoding microRNAs (miRNAs) that have critical roles in the regulation of gene expression.

In this study, we aimed to predict potential mechanisms of bladder cancer related miRNAs and target genes by bioinformatics analyses.

Methods: Here we used the method of text mining to identify nine miRNAs in bladder cancer and adopted protein-protein interaction analysis to identify interaction sites between these miRNAs and related-target genes.

Results: There are two relationship types between bladder cancer and its related miRNAs: causal and unspecified. The Kyoto Encyclopedia of Genes and Genomes (KEGG) enrichment test showed that there were three pathways related to four miRNA targeted genes. The remaining five miRNAs annotated to disease are not enriched in the KEGG pathways. Of these, PIK3R1 is the overlapping gene among 38 genes in the cancer and bladder cancer pathways.
\end{abstract}

Conclusions: These findings provide new insights into the role of miRNAs in the pathway of cancer and give us a hypothesis that miR-127 might play a similar role in regulation and control of PIK3R1.

Keywords: Bladder cancer, miRNA, phosphoinositide 3-kinase

\section{Background}

Bladder cancer is the 10th most common cancer worldwide, with the highest rates reported in Europe, North America and Australia compared to Eastern countries [1,2]; there are an estimated 261,000 new cases diagnosed and 115,000 deaths reported each year. The incidence of bladder cancer is highly correlated with increasing age. It rarely occurs before the age of 40 to 50 years of age, arising most commonly in the seventh decade of life [3,4]. The median ages at diagnosis are 69 years for men and 71 for women [5].

MicroRNAs (miRNAs) are a class of 17 to 27 nucleotide single-stranded RNA molecules that regulate gene

\footnotetext{
* Correspondence: xuyahong@medmail.com.cn

'Department of Urology, the 452nd Hospital of People's Liberation Army, Chengdu 610021, China

Full list of author information is available at the end of the article
}

expression post-transcriptionally. A large body of evidence implicates aberrant miRNA expression patterns in most, if not all, human malignancies. Indeed, cancer is the result of a complex multistep process that involves the accumulation of sequential alterations of several genes and deregulation of those encoding miRNAs [6]. An increasing number of studies have demonstrated that microRNAs can function as potential oncogenes or oncosuppressor genes, depending on the cellular context and the target genes they regulate [7]. The first evidence of alterations of microRNA genes in human cancer came from studies of chronic lymphocytic leukemia (CLL). In a large study of indolent versus aggressive CLL, Calin et al. discovered a signature of 13 microRNAs capable of distinguishing between indolent and aggressive CLL [8]. For bladder cancer, Takahiro et al. demonstrated that KRT7 mRNA was significantly down-regulated by transfection of

\section{Biomed Central}


miR-30-3p, miR-133a and miR-199a in the bladder cancer cell line (KK47), suggesting that these three miRNAs may have a tumor suppressive role via the mechanism underlying transcriptional repression of KRT7 [9].

miRNAs and their target genes are usually validated by quantitative real time polymer transcriptase chain reaction (q-RT-PCR) and Western blot in a wet lab. However, wet lab works consumes a large amount of time and may not be able to obtain the desired results. Hence, we used text mining to identify nine miRNAs in bladder cancer and adopted protein-protein interaction analysis to identify interaction sites between these miRNAs and target genes. We obtained a long list of statistically significant genes without any unifying biological theme. Functional annotation of differentially expressed genes is a necessary and critical step in the analysis of microarray data [10]. A more judicious approach offers query-based access to an integrated database that disseminates biologically rich information across large datasets and displays graphic summaries of functional information. Therefore, we hope to find the important genes that are highly associated with the biological progression of bladder cancer through the use of bioinformatics tools.

\section{Methods}

\section{miRNAs in bladder cancer}

Bladder cancer related miRNAs were drawn from miR2Disease (http://www.mir2disease.org), which is a manually curated database providing a comprehensive resource of microRNA deregulation in various human diseases [11]. The current version of miR2Disease documents 1,939 curated relationships between 299 human microRNAs and 94 human diseases by reviewing more than 600 published papers.

\section{Known targets of miRNA}

TarBase database houses a manually curated collection of experimentally tested miRNA targets in human/ mouse, fruit fly, worm, and zebra fish, distinguishing between those that tested positive and those that tested negative [12]. A search for experimentally proven targets of nine bladder cancer-related miRNAs was performed using the TarBase database (http://diana. cslab.ece.ntua.gr/tarbase) (approved by the 452nd Hospital of People's Liberation Army).

\section{KEGG pathway analysis}

The Kyoto Encyclopedia of Genes and Genomes (KEGG) pathway enrichment was determined by the Fisher exact test with a $P$-value less than 0.05 . The KEGG pathway reveals that most target genes of miRNAs are located in the bladder cancer pathway including the Wnt pathway and cancer pathway. Based on this data, microRNAregulated gene networks associated with bladder cancer
Table 1 Methods and resources for microRNA (miRNA) target prediction

\begin{tabular}{llll}
\hline Method & Type of method & References & Resource \\
\hline TargetScan & $\begin{array}{l}\text { seed } \\
\text { complementarity }\end{array}$ & {$[15]$} & www.targetscan.org \\
miRanda & complementarity & {$[17]$} & www.microrna.org \\
StarBase & CLIP-Seq & {$[16]$} & www.starbase.sysu.edu.cn \\
miRDB & thermodynamics $^{a}$ & {$[18]$} & www.mirdb.org \\
\hline
\end{tabular}

were visualized by Cytoscape [13]. Cytoscape is an open source bioinformatics software platform for visualizing molecular interaction networks and integrating them with gene expression profiles and other state data. Only four miRNAs, including oncomiR miR-21, miR-101-3p, miR221-3p and miR-133 were found in the miRNA-target genes network in this study. Among the remaining five miRNAs, miR-127 is usually differentially expressed as part of a miRNA cluster between normal cells and cancer cells [14]. Hence, we tried to study the functional mechanism of miR-127 in bladder cancer.

\section{Target genes prediction}

TargetScan is a well-known software for predicting microRNA targets from conserved UTR sequences [15], including software (miRanda) and database (microRNA.org). Additionally, starBase is a public platform for exploring miRNA-target interactions from CLIP-Seq (HITS-CLIP, PAR-CLIP) and degradome sequencing (PARE) data [16]. It also provides intersections of multiple target predictions, such as TargetScan, PicTar, miRanda, PITA, RNA22 and miRSVR. In this study, miRNA target genes were obtained from TargetScan (www.targetscan.org), miRanda (www. microrna.org), starBase (www.starbase.sysu.edu.cn) and miRDB (www.mirdb.org), respectively (Table 1). The position of the target site was set in the 3'UTR, with the context score -0.3 .

Table 2 Bladder cancer related microRNAs (miRNAs) in miR2Disease

\begin{tabular}{lll}
\hline miRNA & Reference & Year \\
\hline hsa-miR-199a & {$[9]$} & 2009 \\
hsa-miR-143 & {$[21]$} & 2009 \\
hsa-miR-127 & {$[14]$} & 2006 \\
hsa-miR-30-3p & {$[9]$} & 2009 \\
hsa-miR-221 & {$[22]$} & 2009 \\
hsa-miR-21 & {$[23]$} & 2009 \\
hsa-miR-101 & {$[24]$} & 2009 \\
hsa-miR-129 & {$[25]$} & 2009 \\
hsa-miR-133a & {$[9]$} & 2009 \\
\hline
\end{tabular}




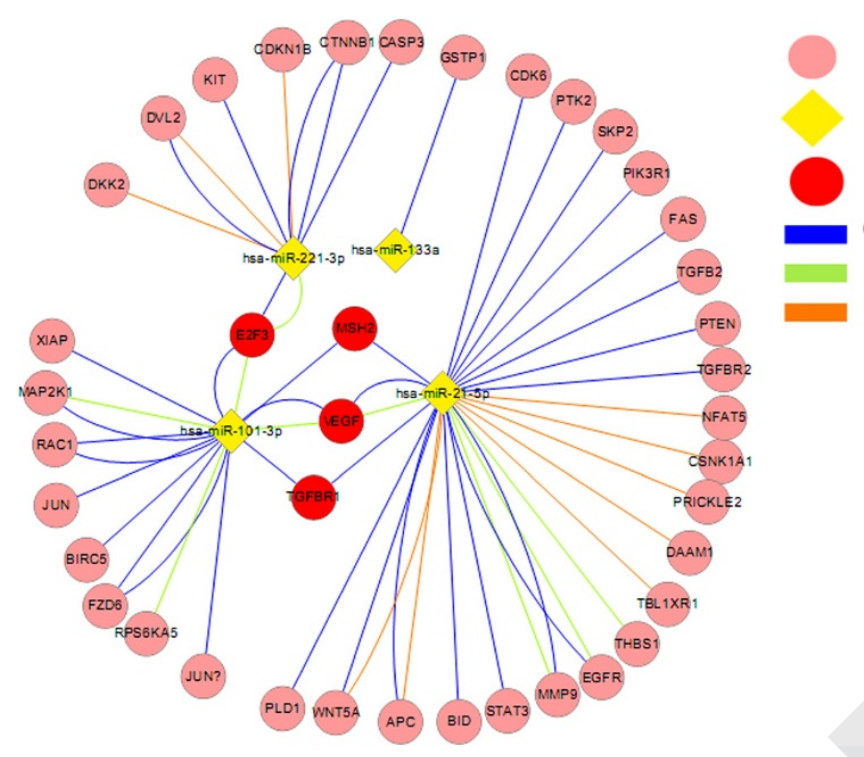

\section{Genes}

miRNAs

Hub Genes

Genes in Cancer pathway

Wnt pathway

Bladder cancer pathway

Figure 1 MicroRNA (miRNA) to mRNA network visualized by Cytoscape. Pink dot represents target genes and red dot represents hub genes; yellow rectangle is miRNA; blue edge indicates target gene located in the pathway of human cancer; green edge means that target gene is located in the Wnt signaling pathway and orange edge that target gene is located in the bladder cancer pathway.

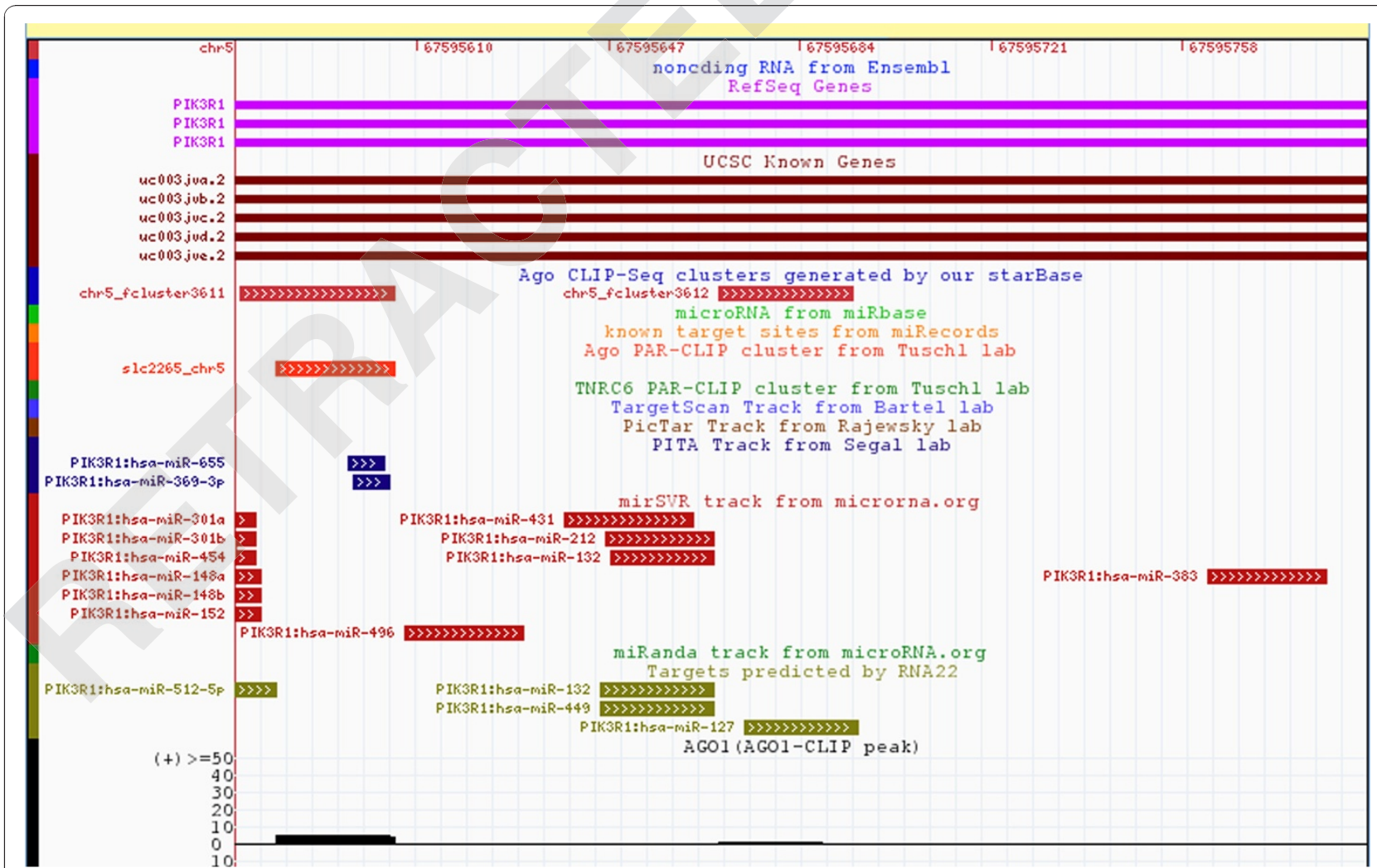

Figure 2 Illustrative screen shots from the deepView browser. The deepView browser provides an integrated view of CLIP-Seq data, known and predicted microRNA (miRNA) target sites, protein-coding genes, non-coding (ncRNA) genes, miRNAs, strand-specific peak clusters, genomewide target-peaks and target-plots. 


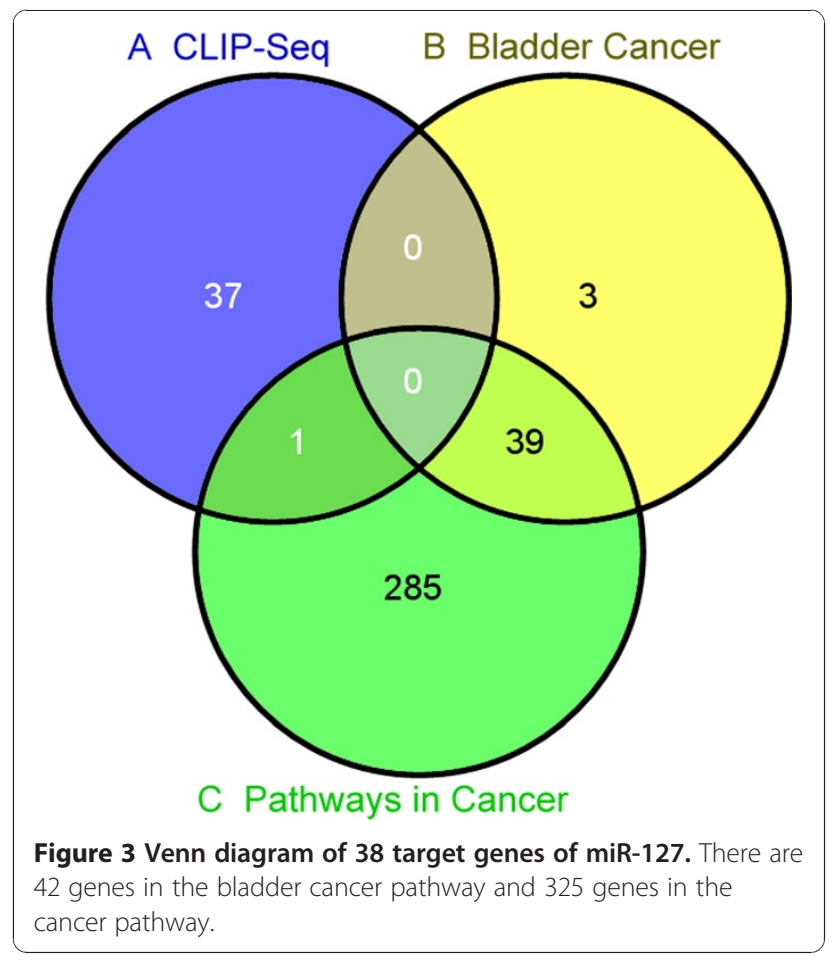

\section{Secondary structures of miRNA binding sites}

We analyzed miRNA targets based on the common criteria, including seed-pairing, free energy of miRNA: target duplex, and proper dynamic programing score. To avoid the incongruency brought about by different standards or outcomes of RNA secondary structure and free energy predicted by distinct algorithms, we recalculated the secondary structures of miRNA. Of note, microRNA-response elements (MREs) involved in duplexes and their free energy for all predicted miRNAs were obtained from the online database by RNAcofold, which is a program for the prediction of hybrid structures of two RNA sequences (www.tbi. univie.ac.at/ivo/RNA) [19,20]. Thus, we provided a relatively equal platform or prerequisite to compare the structure of all these microRNA targets.

\section{Results}

\section{Bladder cancer related miRNAs}

There are two relationship types between bladder cancer and its related miRNAs: causal and unspecified. In this study, data were filtered by selecting causal relationships between bladder cancer and microRNA. Of note, nine miRNAs such as hsa-miR-199a*, hsamiR-143, hsa-miR-127, hsa-miR-30-3p, hsa-miR-221, hsa-miR-21, hsa-miR-101, hsa-miR-129 and hsa-miR-133a were listed (Table 2).

\section{Target genes of nine miRNAs and their roles in the KEGG pathway}

Target genes of nine selected miRNAs were obtained from TargetScan and starBase databases. Then, KEGG pathway analysis was applied to demonstrate the potential biological function of these target genes. Figure 1 shows that target genes of four miRNAs such as hsa-miR-221, hsa-

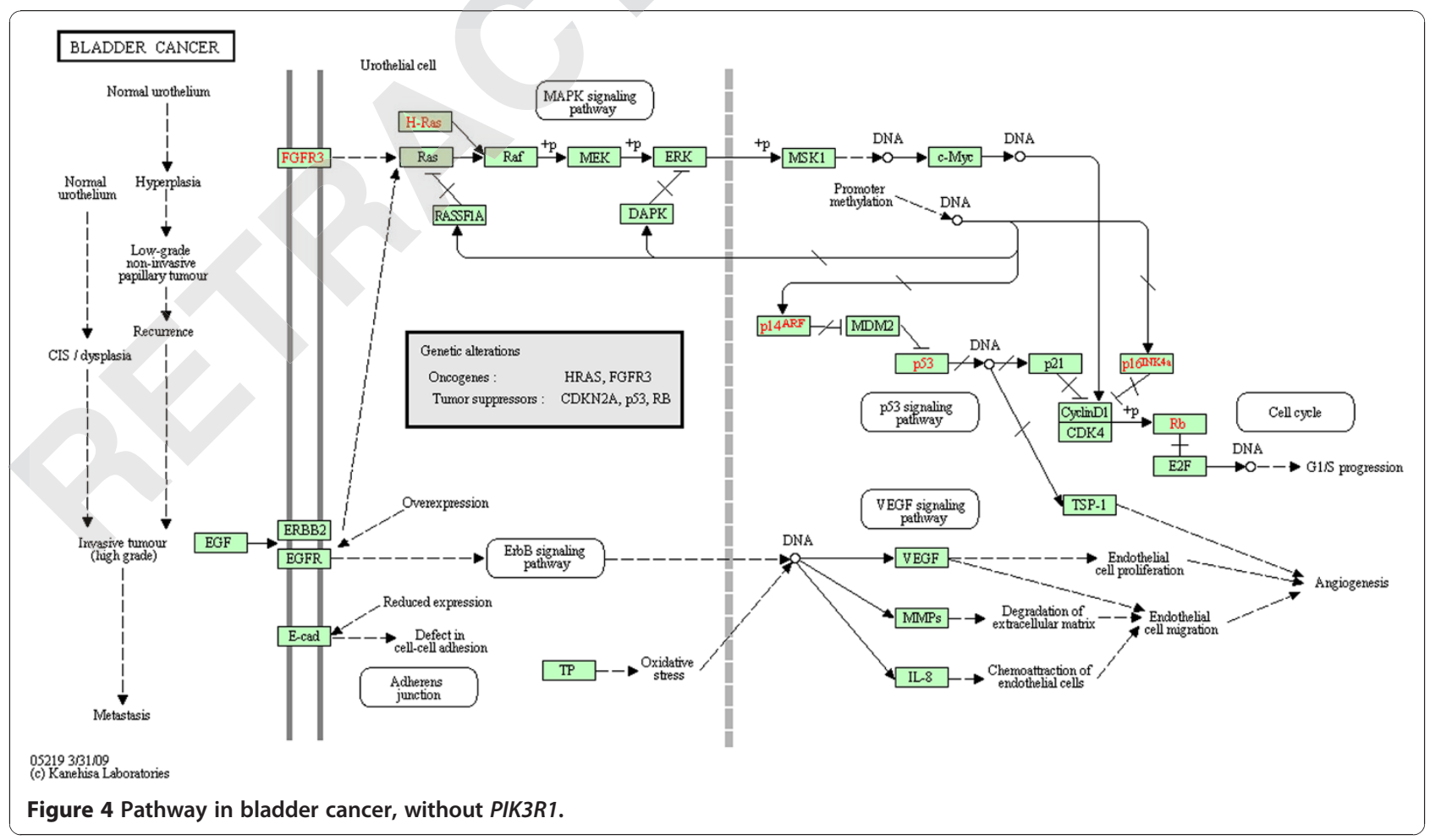




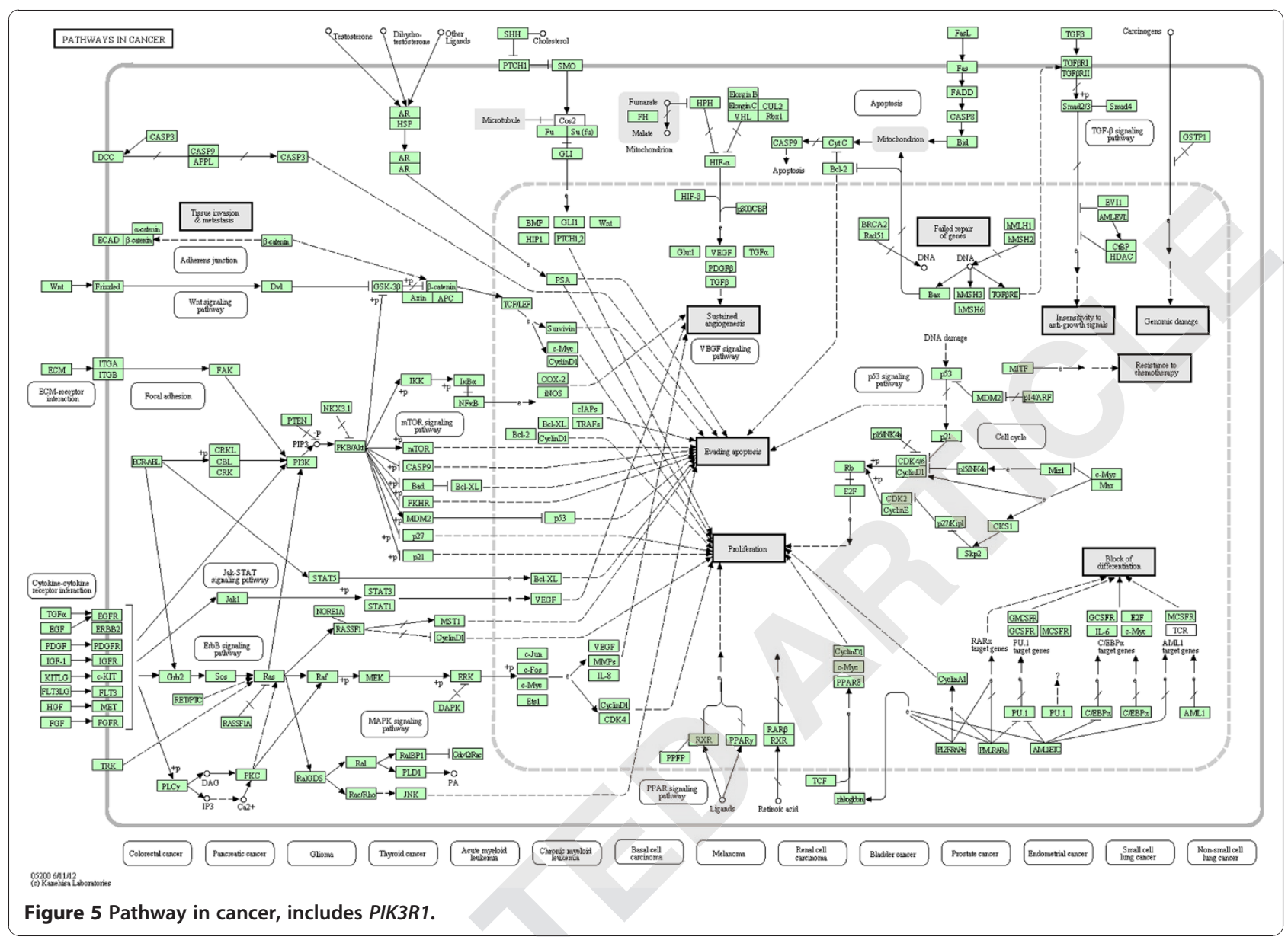

miR-30-3p, hsa-miR-133a and hsa-miR-21 were enriched in three pathways. The remaining five miRNAs annotated to the disease were not enriched in the KEGG pathways.

\section{Target prediction of miR-127}

Given that miR-127 is highly embedded in a CpG island and numerous recent studies have revealed the role of miR-127 in human cancers $[14,26]$, we tried to focus on the study of target prediction of miR-127. Thirty eight target genes regulated by miR-127 were identified via five predicted software instruments including TargetScan, PicTar, PITA, miRanda and RNA22. We also identify the binding site of miR-127 and PIK3R1 in chromosome 5:6759567267595693[+] (GRCh37/hg19) via CLIP-Seq datasets in the deepView genome browser (Figure 2).

In Figure 3, we compared the 38 predicted genes with cancer/bladder cancer related genes and then found that PIK3R1 is the only one gene which overlapped among 38 genes in the bladder cancer pathway (Figure 4) and pathway of cancer (Figure 5). Therefore, we tried to describe miR-127 and its target gene PIK3R1 and their mechanism of action in bladder cancer.
The analysis of miRNA targets was based on the common criteria, including seed-pairing, free energy of miRNA, target duplex, and proper dynamic programing score. To avoid the incongruency brought about by different standards or outcomes of RNA secondary structure and free energy predicted by distinct algorithms, we recalculated the secondary structures of miRNA. MicroRNA-response element (MRE) duplexes and their free energy for all the predicted miRNAs were obtained from the online database by RNAcofold. The four most probably binding sites included SNP-56, -2236, -2611 and -3496 . Additional file 1: Table S3.

\section{Discussion}

We selected known targets of the nine miRNAs and demonstrated their roles in biological process via KEGG pathway analysis. The KEGG enrichment test showed three pathways to be related to the target genes of the four miRNAs. Only four miRNAs, including miR-21, miR-101-3p, miR-221-3p and miR-133 were in the miRNA-target genes network. MiR-127, one of the remaining five miRNAs, is usually differentially expressed as part of a miRNA cluster between normal cells and 
cancer cells [14]. Moreover, a predicted target of miR-127, proto-oncogene BCL6, was down-regulated after treatment with chromatin-modifying drugs [14]. In the current study, we focused on the study of the gene PIK3R1 which was the only overlapping gene among 38 genes in the pathway of cancer.

PIK3R1, also known as GRB1, p85 $\alpha$, p85-ALPHA, is one of the core members involving the phosphoinositide 3kinase (PI3K) pathway [27]. PI3K plays a pivotal role in cell growth, proliferation and survival and inter-signaling systems via this pathway are up-regulated in many types of cancer $[28,29]$. It is strongly hypothesized that alterations of several pathway components can affect the normal function of the PI3K pathway. Knowles et al. has identified that alterations in pathway components PIK3CA, PTEN, AKT1 and TSC1 in bladder cancer are significantly related to tumor phenotype and clinical behavior [30]. PIK3R1 constitutively binds and inhibits the release of catalytic subunit p110 of PI3K. Mutation of PIK3R1 has been observed in ovarian and colon cancer [31], and higher kinase activity was detected in breast cancer [32]. Our findings confirm that the role of PIK3R1 can also be extrapolated in the biological process of bladder cancer.

However, no studies have investigated the role of genetic variations in this pathway in bladder cancer. In this project, we used a large case control study to evaluate the associations of a comprehensive catalog of single nucleotide polymorphisms (SNPs) in the PI3K pathway. Four binding sites of hsa-miR-127-3p including SNP-56, $-2236,-2611$ and -3496 were identified in the $3^{\prime}$ untranslated region of PIK3R1 mRNA, suggesting that single SNPs located at miRNA-binding sites are likely to affect the expression of their targets and might contribute to the pathogenesis of bladder cancer.

\section{Conclusions}

Our data demonstrate a significant association between miR-127 and its target gene of PIK3R1 via analysis of the CLIP-Seq data, RNA secondary structure and free energy. The results indicate that miR-127 plays an important role in regulating PIK3R1 that is involved in both the cancer and bladder cancer pathways.

\section{Additional file}

Additional file 1: Table S3. Binding sits of hsa-miR-127-3p.

\section{Abbreviations \\ (miRNAs): microRNAs; (KEGG): Kyoto encyclopedia of genes and genomes; (CLL): Chronic lymphocytic leukemia; (MREs): microRNA-response elements;} (MRE): MicroRNA-response elements; (SNPs): Single nucleotide polymorphisms.

\section{Competing interests}

The authors declare that they have no competing interests.

\section{Authors' contributions}

$\mathrm{SL}, \mathrm{YL}$ and $J \mathrm{~L}$ collected the nine miRNAs related to bladder cancer in TarBase and participated in KEGG pathway analysis. YL, ZhJ and PC carried out target genes prediction. YG, QZ, XM participated in analysis of miRNA targets based on the common criteria. YX and MY participated in the design of the study and helped to draft the manuscript. All authors read and approved the final manuscript.

\section{Author details}

'Department of Urology, the 452nd Hospital of People's Liberation Army, Chengdu 610021, China. ${ }^{2}$ Cadre aircrew division, the 452nd Hospital of People's Liberation Army, Chengdu 610021, China. ${ }^{3}$ Nursing Department, the 452nd Hospital of People's Liberation Army, Chengdu 610021, China.

Received: 22 January 2013 Accepted: 9 July 2013

Published: 1 September 2013

\section{References}

1. Larsson SC, Andersson SO, Johansson JE, Wolk A: Diabetes mellitus, body size and bladder cancer risk in a prospective study of Swedish men. Eur J Cancer 2008, 44:2655-2660.

2. Meliker J, Nriagu J: Arsenic in drinking water and bladder cancer: review of epidemiological evidence. Trace Metals other Contaminants Environment 2007, 9:551-584

3. Zeegers MPA, Kellen E, Buntinx F, Brandt PA: The association between smoking, beverage consumption, diet and bladder cancer: a systematic literature review. World J Urol 2004, 21:392-401.

4. Shariat SF, Milowsky M, Droller MJ: Bladder cancer in the elderly. Elsevier; 2009:653-667.

5. Volanis D, Kadiyska T, Galanis A, Delakas D, Logotheti S, Zoumpourlis V: Environmental factors and genetic susceptibility promote urinary bladder cancer. Toxicology letters 2010, 193:131-137.

6. Visone R, Croce CM: MiRNAs and cancer. Am J Pathol 2009, 174:1131.

7. Iorio MV, Croce CM: MicroRNAs in cancer: small molecules with a huge impact. J Clin Oncol 2009, 27:5848-5856.

8. Calin GA, Ferracin M, Cimmino A, Di Leva G, Shimizu M, Wojcik SE, lorio MV, Visone $\mathrm{R}$, Sever NI, Fabbri M: A microRNA signature associated with prognosis and progression in chronic lymphocytic leukemia. New Engl $J$ Med 2005, 353:1793-1801.

9. Ichimi T, Enokida H, Okuno Y, Kunimoto R, Chiyomaru T, Kawamoto K, Kawahara K, Toki K, Kawakami K, Nishiyama K: Identification of novel microRNA targets based on microRNA signatures in bladder cancer. Int J Cancer 2009, 125:345-352.

10. Dennis G Jr, Sherman BT, Hosack DA, Yang J, Gao W, Lane HC, Lempicki RA: DAVID: database for annotation, visualization, and integrated discovery. Genome Biol 2003, 4:P3.

11. Jiang Q, Wang Y, Hao Y, Juan L, Teng M, Zhang X, Li M, Wang G, Liu Y miR2Disease: a manually curated database for microRNA deregulation in human disease. Nucleic acids res 2009, 37:D98-D104.

12. Sethupathy P, Corda B, Hatzigeorgiou AG: TarBase: a comprehensive database of experimentally supported animal microRNA targets. Rna 2006, 12:192-197.

13. Shannon P, Markiel A, Ozier O, Baliga NS, Wang JT, Ramage D, Amin N, Schwikowski B, Ideker T: Cytoscape: a software environment for integrated models of biomolecular interaction networks. Genome res 2003, 13:2498-2504.

14. Saito Y, Liang G, Egger G, Friedman JM, Chuang JC, Coetzee GA, Jones PA: Specific activation of microRNA-127 with downregulation of the protooncogene BCL6by chromatin-modifying drugs in human cancer cells. Cancer Cell 2006, 9:435-443.

15. Lewis BP, Burge CB, Bartel DP: Conserved seed pairing, often flanked by adenosines, indicates that thousands of human genes are microRNA targets. Cell 2005, 120:15-20.

16. Yang JH, Li JH, Shao P, Zhou H, Chen YQ, Qu LH: starBase: a database for exploring microRNA-mRNA interaction maps from Argonaute CLIP-Seq and Degradome-Seq data. Nucleic acids res 2011, 39:D202-D209.

17. Betel D, Koppal A, Agius P, Sander C, Leslie C: Comprehensive modeling of microRNA targets predicts functional non-conserved and non-canonical sites. Genome biology 2010, 11:R90.

18. Wang $X$, El Naqa IM: Prediction of both conserved and nonconserved microRNA targets in animals. Bioinformatics 2008, 24:325-332. 
19. Hofacker IL: Vienna RNA secondary structure server. Nucleic acids res 2003, 31:3429-3431.

20. Bernhart SH, Tafer H, Mückstein U, Flamm C, Stadler PF, Hofacker IL: Partition function and base pairing probabilities of RNA heterodimers. Algorithms for Molecular Biology 2006, 1:3.

21. Lin T, Dong W, Huang J, Pan Q, Fan X, Zhang C, Huang L: MicroRNA-143 as a tumor suppressor for bladder cancer. The J urology 2009, 181:1372-1380.

22. Lu Q, Lu C, Zhou GP, Zhang W, Xiao H, Wang XR: MicroRNA-221 silencing predisposed human bladder cancer cells to undergo apoptosis induced by TRAIL. Elsevier; 2010:635-641.

23. Catto JWF, Miah S, Owen HC, Bryant $H$, Myers K, Dudziec E, Larré S, Milo M, Rehman I, Rosario DJ: Distinct microRNA alterations characterize high-and low-grade bladder cancer. Cancer res 2009, 69:8472.

24. Friedman JM, Liang G, Liu CC, Wolff EM, Tsai YC, Ye W, Zhou X, Jones PA: The putative tumor suppressor microRNA-101 modulates the cancer epigenome by repressing the polycomb group protein EZH2. Cancer res 2009, 69:2623.

25. Dyrskjøt L, Ostenfeld MS, Bramsen JB, Silahtaroglu AN, Lamy P, Ramanathan R, Fristrup N, Jensen JL, Andersen CL, Zieger K: Genomic profiling of microRNAs in bladder cancer: miR-129 is associated with poor outcome and promotes cell death in vitro. Cancer res 2009, 69:4851.

26. Lu J, Getz G, Miska EA, Alvarez-Saavedra E, Lamb J, Peck D, Sweet-Cordero A, Ebert BL, Mak RH, Ferrando AA: MicroRNA expression profiles classify human cancers. Nature 2005, 435:834-838.

27. Fruman DA, Cantley LC, Carpenter CL: Structural organization and alternative splicing of the murine phosphoinositide 3-kinase p85 (alpha) gene. Genomics 1996, 37:113-121.

28. Cantley LC: The phosphoinositide 3-kinase pathway. Science Signaling 2002, 296:1655.

29. Luo J, Manning BD, Cantley LC: Targeting the PI3K-Akt pathway in human cancer: rationale and promise. Cancer Cell 2003, 4:257.

30. Knowles MA, Platt FM, Ross RL, Hurst CD: Phosphatidylinositol 3-kinase (PI3K) pathway activation in bladder cancer. Cancer and Metastasis Reviews 2009, 28:305-316.

31. Terauchi Y, Tsuji Y, Satoh S, Minoura H, Murakami K, Okuno A, Inukai K Asano T, Kaburagi Y, Ueki K: Increased insulin sensitivity and hypoglycaemia in mice lacking the p85a subunit of phosphoinositide 3-kinase. Nature Genetics 1999, 21:230-235.

32. Sun M, Paciga JE, Feldman RI, Yuan Z-q, Coppola D, Lu YY, Shelley SA, Nicosia SV, Cheng JQ: Phosphatidylinositol-3-OH kinase (PI3K)/AKT2, activated in breast cancer, regulates and is induced by estrogen receptor a (ERa) via interaction between ERa and PI3K. Cancer res 2001, 61:5985-5991.

\section{doi:10.1186/2047-783X-18-29}

Cite this article as: $\mathrm{Xu}$ et al:: Integrated gene network analysis and text mining revealing PIK3R1 regulated by miR-127 in human bladder cancer. European Journal of Medical Research 2013 18:29.

\section{Submit your next manuscript to BioMed Central and take full advantage of:}

- Convenient online submission

- Thorough peer review

- No space constraints or color figure charges

- Immediate publication on acceptance

- Inclusion in PubMed, CAS, Scopus and Google Scholar

- Research which is freely available for redistribution 